\title{
TCP PERFORMANCES IN A HYBRID BROADCAST/TELECOMMUNICATION SYSTEM
}

\author{
Davy Darche ${ }^{1}$, Francis Lepage ${ }^{2}$, Eric Gnaedinger ${ }^{3}$ \\ TDF 1, rue Marconi $57000 \mathrm{Metz}^{1}$, CRAN, CNRS UMR 7039, Université Henri Poincaré NANCY \\ I BP 23954506 Vandoeuvre-les-Nancy Cedex, FRANCE ${ }^{2,3}$ \\ Email: davy.darche@ tdf.fr $^{1}$,francis.lepage@cran.uhp-nancy.fr ${ }^{2}$,eric.gnaedinger@cran.uhp-nancy.fr ${ }^{3}$
}

\begin{abstract}
High speed cable/ADSL connections are rapidly becoming the standard for Internet at home. Conversely, mobile network operators only offer low bit rate data services to their $2.5 \mathrm{G}$ users, and $3 \mathrm{G}$ will not be in full swing for another couple of years. In this context, the cooperation between a mobile telecommunication network and a broadcast network can be a suitable alternative to enhance this offer with high speed e-mail, web browsing, file download or even peer-to-peer services. This paper presents a network architecture based on the coupling of a GPRS uplink with a DVB-T downlink, to provide Internet connectivity for unicast and multicast services. We first study the GPRS network and show the issues raised by specific use of this return channel. Then, we analyse, using simulations, how to tune the TCP parameters to increase the GPRS/DVB-T hybrid network performances. Finally, we describe the network architecture of the deployed system, and evaluate its performances.
\end{abstract}

Keywords: Hybrid network, cooperation, asymmetry, TCP, GPRS, DVB-T

\section{Introduction}

In a hybrid network, the uplink and downlink are different. In our case we use DVB-T (Digital Video Broadcasting Terrestrial) for the downlink and GPRS (Generalised Packet Radio Services) for the uplink. The request (Req.) is sent by GPRS to the HNIS (Hybrid Network Interconnection System). Then the request is forwarded to the appropriate server on the Internet, whose reply is routed on the DVB-T network [1].

DVB-T is a robust, unidirectional, broadcast network with high bandwidth, whereas GPRS is a bidirectional telecommunication network, with a low bandwidth, and a considerable bit error rate [2]. The cooperation between these two networks creates important asymmetries between the two links of communication as shown by experimental measures (Tab. 1). Thereafter we will study the impact of this asymmetry on the TCP mechanisms. Indeed, the TCP pro- 


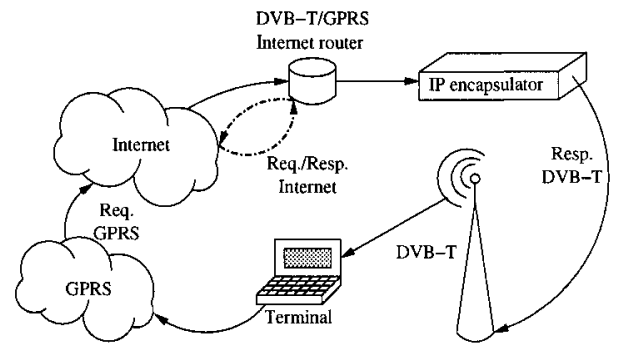

Figure 1. Hybrid network architecture

tocol, without specific tuning, has poor performances in this hybrid network architecture (Fig. 1).

\begin{tabular}{lcr}
\hline QoS parameters & GPRS (uplink) & $D V B-T$ \\
\hline Latency & $700 \mathrm{~ms}-4 \mathrm{~s}$ & $50-60 \mathrm{~ms}$ \\
Jitter & $4 \mathrm{~s}$ & $10 \mathrm{~ms}$ \\
Packet error rate & $1-2 \%$ & $<10^{-6}$ \\
Available bandwidth & $20 \mathrm{kbits} . \mathrm{s}^{-1}$ & $20 \mathrm{Mbits}^{-1}$ \\
\hline
\end{tabular}

Table 1. GPRS/DVB-T QoS parameters

Measures in table (Tab. 1) are performed for several bit rates (0.6kbits.s ${ }^{-1}$ to full bandwidth).

\section{Issues raised by the GPRS return channel}

In this section we study the GPRS return channel issues. The following experimentations are made on real GPRS networks. We use several GPRS subscriptions of Finnish and French operators. All the results being very similar with the different operators, we only analyse the test results of one operator, RADIOLINJA. All the GPRS subscriptions have two timeslots for upload. The experimental tests consist of sending IP packets (ICMP packet, 84 bytes) from a terminal to a server, and receiving these packets. The sent packets generate a constant bit rate. Then we study the RTT (Round Trip Time) related to each packet. In one case the packets are sent by GPRS and are received by GPRS. We call this transmission mode bidirectional, since we use the uplink and the downlink of GPRS. In another case, the packets are sent by GPRS and are received by LAN. We call this transmission mode unidirectional. As the latency of the transmission on the LAN is very low compared to the latency on GPRS (LAN $<1 \mathrm{~ms}$, GPRS $\simeq 1 \mathrm{~s}$ ), we do not take into account the latency of the LAN. 
So, we can evaluate the behavior of the GPRS latency as a function of the uplink load, and thus the consequences of an exclusive use of the GPRS uplink.

\subsection{GPRS Bidirectional mode}

We can notice in the figure 2 that the latency of the GPRS network is around $1 \mathrm{~s}$ (despite many oscillations), for throughputs ranging from $5 \mathrm{kbits} . \mathrm{s}^{-1}$ to $20 \mathrm{kbits} . \mathrm{s}^{-1}$. At $25 \mathrm{kbits} . \mathrm{s}^{-1}$, we reach the bandwidth limit of the GPRS connection. The latency increases strongly due to buffer mechanisms in the GPRS network.

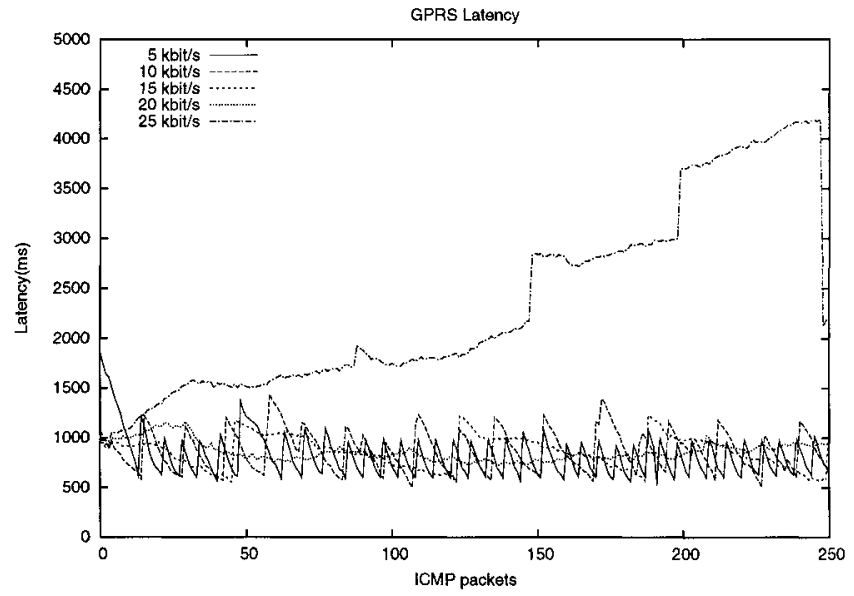

Figure 2. GPRS latency in bidirectional mode

For lower bit rates, the throughput oscillates presumably because of the time division of the bandwidth in the transmission mode of GPRS.

\subsection{GPRS Unidirectional mode}

We note that the behavior of the GPRS network (latency according to the load of the uplink) in the unidirectional mode (Fig. 3) is totally different from the bidirectional mode. The latency increases faster for small throughputs. A detailed analysis of the GPRS latencies shows a brutal jump from $2.4 \mathrm{~s}$ to $4 \mathrm{~s}$ for the traffic of $15 \mathrm{kbits} . \mathrm{s}^{-1}, 20 \mathrm{kbits} . \mathrm{s}^{-1}, 25 \mathrm{kbits} . \mathrm{s}^{-1}$. The latency increases quasi-linearly until reaching a critical threshold of $2.4 \mathrm{~s}$ (Fig. 3). There are therefore two different behaviors of the GPRS latency:

- A stationary, oscillating mode for throughput lower than $10 \mathrm{kbits} . \mathrm{s}^{-1}$.

- A quasi-lineary, increasing mode involving a latency jump from $2.4 \mathrm{~s}$ to $4 \mathrm{~s}$, for throughput greater than $10 \mathrm{kbits}_{\mathrm{s}}{ }^{-1}$. 


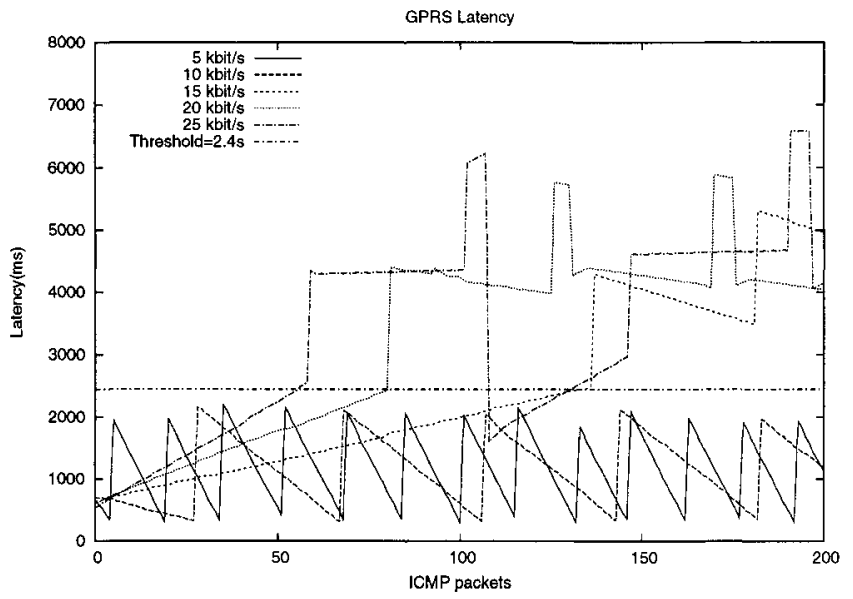

Figure 3. GPRS latency in unidirectional mode

\subsection{GPRS uplink critical throughput}

A more precise study permits to specify the critical throughput $\gamma$ beyond which the latency jumps to $4 \mathrm{~s}$. As shown in previous sections, the GPRS latency varies with the type of use (unidirectional or bidirectional). In our hybrid network architecture only the GPRS uplink is used, the latency of the GPRS network can switch between two differents modes. In one mode the throughput is lower than $\gamma$ and the latency does not exceed 2s. In the other mode, the throughput is greater than $\gamma$ and the latency increases to $4 \mathrm{~s}-5 \mathrm{~s}$.

If we consider the GPRS network as a black box model, we can conclude that the GPRS uplink quality of service is probably related to the traffic on the GPRS downlink. It is therefore necessary to evaluate the throughput needed on the uplink for a specific service when we use GPRS as a return channel. In the GPRS/DVB-T hybrid network, we must limit the uplink traffic to a value smaller than $\gamma$ to prevent the jump of the latency. This brutal increase of the latency can otherwise be considered by TCP as a timeout. We will solve the problem at the transport layer, as we have limited control on the GPRS network.

\section{Simulation studies of the hybrid network performances}

\subsection{Simulation model of the hybrid network}

In this section, we use the NS2 (Network Simulator v2) simulation tool to study the hybrid network architecture, to solve the GPRS return channel issues, and assess the impact of the various asymmetries of this system. We only simulate OSI layers three and above. In particular the encapsulation mechanisms 
inherent to the DVB-T and GPRS transmission are not simulated. DVB-T is represented by a unidirectional link with $20 \mathrm{Mbits.} \mathrm{s}^{-1}$ bandwidth and $50 \mathrm{~ms}$ of delay. GPRS is modeled by a unidirectional link with $20 \mathrm{kbits. \textrm {s } ^ { - 1 }}$ bandwidth and $700 \mathrm{~ms}$ of delay (Fig. 4). We simulate a data transfer from a server $\mathbf{S}$ to a client $\mathbf{C}$ trough a TCP connection (FTP). We add a node $\mathbf{n}$ and a new link to introduce the packet loss rate. This link has the same bandwidth as GPRS and very low delay $(1 \mathrm{~ms})$. The node $\mathbf{n}$ has a very large buffer to simulate the GPRS network buffer whereas the server and the client have minimal buffers.

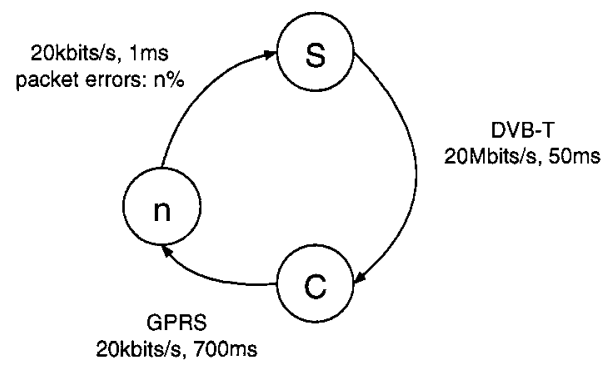

Figure 4. The simulation model

\subsection{Asymmetries}

Asymmetry of delays. The difference of delay between GPRS and DVB$\mathrm{T}$ is very important, with respectively $50 \mathrm{~ms}$ on DVB-T and $700 \mathrm{~ms}$ on GPRS. However, this difference does not affect the TCP functions. It is the large RTT (Round Trip Time), around $750 \mathrm{~ms}$, that hinders the throughput of the TCP communication.

$$
\begin{gathered}
\text { Throughput }_{\max }=\frac{T C P W \text { ndSize }}{R T T} \\
\rho=\frac{R T T_{G P R S}}{R T T_{D V B-T}}
\end{gathered}
$$

For the simulations, the global RTT is fixed to $750 \mathrm{~ms}$ ( $R T T_{G P R S}+$ $R T T_{D V B-T}$ ), and we vary the ratio $\rho$. The ratio $\rho$ has absolutely no effect, the simulation results show that the throughput on the downlink is always $626 \mathrm{kbits}^{-1} \mathrm{~s}^{-}$. However to increase the TCP performances with this large RTT, we have to increase the TCP window sizes.

Bandwidth asymmetry. The maximal size of a packet, that can be sent from the terminal to the server and from the server to the terminal, without fragmentation is 1500 bytes $\left(M T U_{\max }=1500\right)$. The TCP protocol generates a maximum of one Ack (40 bytes) per packet of data. A data transfer from the 
server to the terminal produces, on the uplink, a traffic with a throughput that cannot exceed $2.67 \%$ of the downlink throughput.

$$
\frac{A c k_{s i z e}}{M T U_{\max }}=0.0267
$$

TCP uses a cumulative acknowledgement mechanism, and thus one Ack can acknowledge more than one packet of data. We have always:

$$
\begin{gathered}
\text { number of Ack } n \leq \text { number of data packets } m \\
\frac{n \cdot A c k_{\text {size }}}{m \cdot M T U_{\max }} \leq 0.0267
\end{gathered}
$$

By using the full bandwidth capacity of the uplink (20kbits.s ${ }^{-1}$ ), and without the other constraints (RTT, packet error rate...), we can estimate a minimum throughput of $750 \mathrm{kbits} . \mathrm{s}^{-1}$ for the download traffic. The TCP option Delayed Ack permits to defer the sending of the Acks for a duration $\delta$. We can thus increase the number of data packets acknowledged by one Ack. So, it is possible to reduce the bandwidth used on the uplink, or to increase the throughput on the downlink.

\begin{tabular}{lcr}
\hline TCP option & GPRS throughput & DVB-T throughput \\
\hline- & $19.9 \mathrm{kbits} / \mathrm{s}$ & $750 \mathrm{kbits} / \mathrm{s}$ \\
Delayed Ack $5 \mathrm{~ms}$ & $19.9 \mathrm{kbits} / \mathrm{s}$ & $1200 \mathrm{kbits} / \mathrm{s}$ \\
Delayed Ack $20 \mathrm{~ms}$ & $19.9 \mathrm{kbits} / \mathrm{s}$ & $1500 \mathrm{kbits} / \mathrm{s}$ \\
\hline
\end{tabular}

Table 2. Bandwidth asymmetry, GPRS latency $1 \mathrm{~ms}$, DVB-T latency $1 \mathrm{~ms}$

We have run two series of simulations. In the first series, we do not take into account the temporal dimension of the system by fixing the DVB-T and GPRS latencies to $1 \mathrm{~ms}$ (Tab. 2). We notice that the traffic on the GPRS link uses all the bandwidth capacity (20kbits.s ${ }^{-1}$ ) and the throughput on the downlink increases.

In the second simulation, we set the GPRS latency to $700 \mathrm{~ms}$ and the DVBT latency to $50 \mathrm{~ms}$ and we tune the TCP DelayedAck options (Tab. 3). The throughput of the downlink increases slightly more while the traffic on the uplink decreases strongly. In this case the throughput of the system is limited by the temporal dimension (Expr. 1). The value of the TCP window size is 50MSS (Maximum Segment Size) [4] [6] [5]. One MSS is equal to the MTU without the TCP and IP headers, 1460 bytes. Consequently the maximal theorical throughput is $778.6 \mathrm{kbits} . \mathrm{s}^{-1}$. This theorical value is confirmed by the simulations. By increasing the value of the TCP window size to 100MSS, we can 


\begin{tabular}{lcr}
\hline TCP option & GPRS throughput & DVB-T throughput \\
\hline- & $19.9 \mathrm{kbits} / \mathrm{s}$ & $747.2 \mathrm{kbits} / \mathrm{s}$ \\
Delayed Ack $5 \mathrm{~ms}$ & $12.5 \mathrm{kbits} / \mathrm{s}$ & $757.0 \mathrm{kbits} / \mathrm{s}$ \\
Delayed Ack $10 \mathrm{~ms}$ & $12.4 \mathrm{kbits} / \mathrm{s}$ & $752.3 \mathrm{kbits} / \mathrm{s}$ \\
Delayed Ack $20 \mathrm{~ms}$ & $10.1 \mathrm{kbits} / \mathrm{s}$ & $761.1 \mathrm{kbits} / \mathrm{s}$ \\
\hline
\end{tabular}

Table 3. Bandwidth asymmetry, GPRS latency $700 \mathrm{~ms}$, DVB-T latency $50 \mathrm{~ms}$

theorically double the throughput of the system. Once again the full bandwidth of the uplink is used, and the downlink throughput reaches $1490 \mathrm{kbits. \textrm {s } ^ { - 1 }}$.

The asymmetry of the packet error rate. In most networks, uplink and downlink have the same packet error rate. In this case, the DVB-T communication link has a very low packet error rate, lower than $10^{-6}$, whereas the GPRS link has a large packet error rate, around $1 \%$. By varying the error rate on the two links, we study how these differences affect the TCP performances. We use the standard TCP implementation and options for these simulations.

\begin{tabular}{lcr}
\hline GPRS error rate & DVB-T error rate & Throughput on DVB-T \\
\hline $0 \%, 1 \%, 2 \%$ & $0 \%$ & $749 \mathrm{kbits} / \mathrm{s}$ \\
$0 \%$ & $0.01 \%$ & $707.4 \mathrm{kbits} / \mathrm{s}$ \\
$0 \%$ & $0.1 \%$ & $488.4 \mathrm{kbits} / \mathrm{s}$ \\
$0 \%$ & $1 \%$ & $142.8 \mathrm{kbits} / \mathrm{s}$ \\
$2 \%$ & $0.01 \%$ & $704.6 \mathrm{kbits} / \mathrm{s}$ \\
\hline
\end{tabular}

Table 4. Error rate asymmetry

The simulations show that the hybrid architecture is more sensitive to the errors on the data download link than to the errors on the return channel. This behaviour is inherent to the cumulative aspect of the TCP acknowledgement mechanism. Conversely, when the loss of a data packet occurs, TCP has to wait for a timeout to detect the packet loss and resend it. This timeout is similar to the RTT, $750 \mathrm{~ms}$. This decreases strongly the throughput of the system. The reliability of the DVB-T transmission to transfer the data packet is appropriate for the service we want to provide to the end user (data transfer to the client). Moreover, the error rate of GPRS has very limited influences on the quality of the service. We consider the system in degraded mode, with $2 \%$ of error rate 
on GPRS and $0.01 \%$ of error rate on DVB-T. We compare the different TCP implementations with differents options (Tab. 5).

\begin{tabular}{lcr}
\hline TCP implementation & GPRS throughput & DVB-T throughput \\
\hline Tahoe TCP & $18.7 \mathrm{kbits} / \mathrm{s}$ & $704.6 \mathrm{kbits} / \mathrm{s}$ \\
Tahoe TCP, DelAck & $9.7 \mathrm{kbits} / \mathrm{s}$ & $693.6 \mathrm{kbits} / \mathrm{s}$ \\
Reno TCP & $19.1 \mathrm{kbits} / \mathrm{s}$ & $718.7 \mathrm{kbits} / \mathrm{s}$ \\
Reno TCP, DelAck & $9.9 \mathrm{kbits} / \mathrm{s}$ & $710.2 \mathrm{kbits} / \mathrm{s}$ \\
NewReno TCP & $19.1 \mathrm{kbits} / \mathrm{s}$ & $718.7 \mathrm{kbits} / \mathrm{s}$ \\
NewReno TCP, DelAck & $9.9 \mathrm{kbits} / \mathrm{s}$ & $710.2 \mathrm{kbits} / \mathrm{s}$ \\
Sack TCP & $19.1 \mathrm{kbits} / \mathrm{s}$ & $717.2 \mathrm{kbits} / \mathrm{s}$ \\
Sack TCP, DelAck & $9.7 \mathrm{kbits} / \mathrm{s}$ & $700.6 \mathrm{kbits} / \mathrm{s}$ \\
\hline
\end{tabular}

Table 5. TCP implementation in degraded mode

We notice that the use of the TCP Delayed Ack option slightly decreases the performances. As there are fewer Ack to acknowledge packets with the Delayed Ack option, the loss of one Ack increases the probability to not acknowledge a data packet. However we can see that we only use half of the uplink bandwidth. This way, we can limit the throughput on GPRS below the critical GPRS throughput, 10kbits.s ${ }^{-1}$, and we can solve the GPRS return channel issue.

\subsection{Hybrid routing}

With the delayed ack option, the upload traffic remains under 10kbits.s ${ }^{-1}$. However, if the terminal initiates a data transfer to the server, a new traffic will be added to the $10 \mathrm{kbits} . \mathrm{s}^{-1}$ of acknowledgment traffic. A way to prevent the latency jump on the GPRS link, is to adapt the traffic policy routing on the HNIS router. The Ack related to the upload traffic is routed to the GPRS downlink. Thus the GPRS behaviour switches to a bidirectional mode and the latency is fixed to a value near $700 \mathrm{~ms}$. We use a traffic control module to regulate the data traffic on the downlink and to prevent bursts of traffic. This contributes to enforce the architecture stability. Another functionality is to ensure the bandwidth for a single user.

\section{Experimentations}

For the experimentations, we use a VPN to connect the terminal to the DVBT/GPRS Internet router. The VPN offers the compression and encryption capabilities. We use a VPN connection to pass through firewall and/or NAT router. 
The tests consist of a FTP transfer from the router/server to the terminal. In one case we use the TCP DelayedAck option (set to 600ms) and in the other case the terminal acknowledges all the packets.

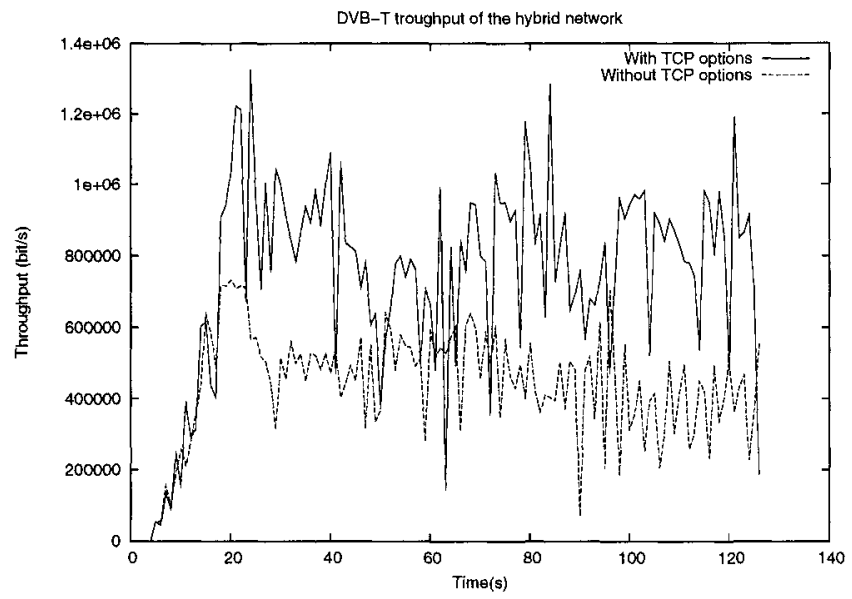

Figure 5. DVB-T throughput in the hybrid architecture

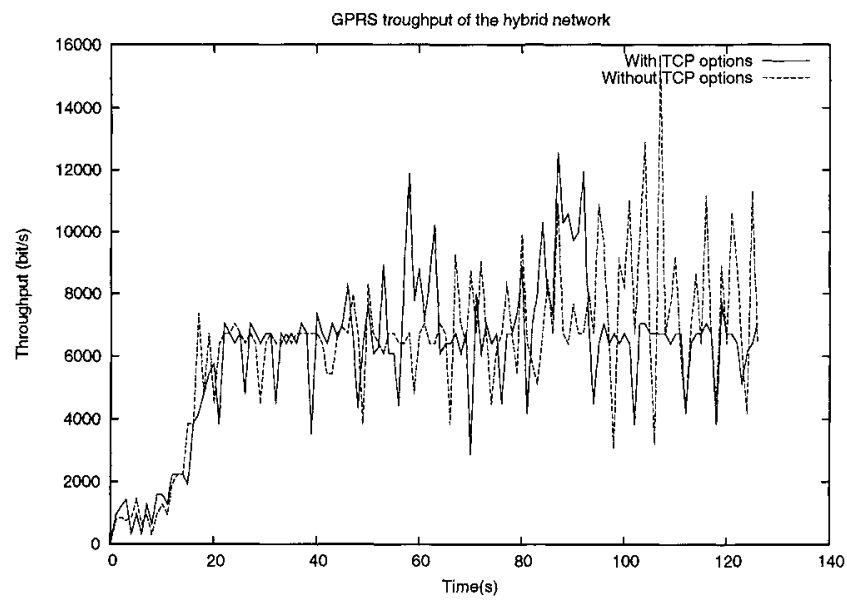

Figure 6. GPRS throughput in the hybrid architecture

We notice that the GPRS throughput is around $6 \mathrm{kbit}^{-1}$ for the two FTP transfers. With the TCP DelayedAck option, the throughput of the DVB-T traffic can reach $1.3 \mathrm{Mbits.s^{-1 }}$ whereas without the option, the throughput is limited to $700 \mathrm{kbits}^{-1}$. The average throughput is $747.8 \mathrm{kbit} . \mathrm{s}^{-1}$ with the TCP 


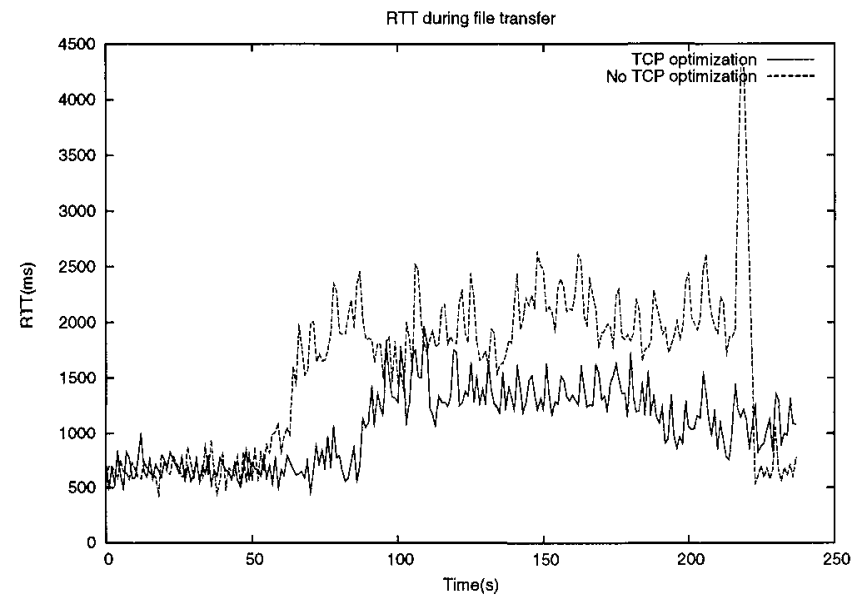

Figure 7. RTT in the hybrid network

option, against $435.3 \mathrm{kbit}^{-1}$ without the TCP DelAck. With the TCP optimizations, the RTT has a value of $1185 \mathrm{~ms}$, whereas without the RTT has an average of $1881 \mathrm{~ms}$ (Fig 7). A low RTT contributes to increase the bit rate (Expr. 1). Not only the adaptation of TCP increases the performances, but it increases the stability of the architecture as well.

\section{Conclusion}

Initially, the TCP protocol was developed to prevent congestion in wired networks, TCP is not adapted for wireless networks, with high bit error rate, high asymmetries and multiple interfaces. However for current Internet services, a reliable transport mechanism such as TCP is required. Due to dynamic resource allocation, the QoS of the GPRS uplink is related to the GPRS downlink traffic. This behavior is a major issue as it impacts the latency in a DVBT/GPRS hybrid network architecture. To solve this issue at the transport level, the TCP/IP stack has been tuned.

- Use of TCP Delayed Ack option to reduce strongly the return channel traffic.

- An advanced policy routing and traffic control to prevent latency peaks during upload data transfer.

With these tunings, on the hybrid network architecture, it becomes possible to provide stable high bit rate services (HTTP, FTP, peers to peers, streaming...) in a wireless environment. 


\section{References}

[1] Hybrid Mobile Interactive Services combining DVB-T and GPRS, 2001, Christian Rauch Vodafone Pilotentwicklung $\mathrm{GmbH}$ (formerly Mannesmann Pilotentwicklung $\mathrm{GmbH}$ ) Wolfgang Kellerer, Peter Sties Munich University of Technology (TUM), Institute of Communication Networks Arcisstr.

[2] Multi-Layer Protocol Tracing in a GPRS Network, 2002, Andrei Gurtov, Matti Passoja, Olli Aalto, Mika Raitola Cellular Systems Development Sonera Helsinki, Finland

[3] W. Richard Stevens TCP/IP Illustrated, Volume 1 Ed. Addison Wesley, 1994 ISBN 0-20163346-9

[4] RFC 793 - Transmission Control Protocol

[5] V. Jacobson, R. Braden and D. Borman, RFC 1323 - TCP Extensions for High Performance.

[6] M. Mathis, J. Mahdavi, S. Floyd and A. Romanow, RFC 2018 - TCP Selective Acknowledgement Options 